\title{
The design and implementation of the surveillance and control system for photovoltaic power station
}

\author{
Wang Jian,Song Shuting,Zhang Yong \\ The Department of information technology \\ Shanxi Electric Power Corporation \\ Taiyuan,China \\ tywangjian@vip.sina.com
}

\author{
Gao Feng \\ Smart Grid Dep \\ Huada Tianyuan (Beijing) Electric Porwer Tecnaology \\ Co., LTD \\ Beijing, China \\ gaofeng6666@126.com
}

\begin{abstract}
According to the characteristics of PV power station, how to present a new intelligent surveillance and control system of PV power station, which collects the operating parameters at real-time and control the running status of the $P V$ power station through wireless networks and the Internet, to guarantee the security, stability and economic operation of grid-connected PV system, becomes a problem which electric power enterprises have to be solved urgently. Based on the analysis of several remote monitoring technology projects which are general practical in PV system, the article designs a set of photovoltaic power plant monitoring system aimed at the security, stability of grid-connected PV system, which is described from the ideas of construction, software architecture, and development of hardware and so on.
\end{abstract}

Keywords-photovoltaic power station; monitoring; gridconnected PV system; power grid

\section{INTRODUCTION}

As the greenhouse effect has made the environment in the earth increasingly worse, human have become increasingly concerned with the issue of energy, which is an important part of the problems of the environment. With advantages such as non-polluting, inexhaustible, etc. Solar power has become the alternative energy resource in a lot of fields which is more economical and more attractive in terms of technology when compared to traditional energy sources. For example, it can be applied to various types of microwave transmission station, wireless launch points, hydrological monitoring stations and so on. Grid-connected PV systems generally consist of solar photovoltaic panels, inverters and AC controller. A lot of parameters should to be measured, preserved, analyzed, and controlled to ensure that each device can function properly.

However, the present photovoltaic power station has some general problems as follows:

1) As we all know, daily operating data has important reference value to the management of power plant systems, equipment maintenance, and future research and analysis. However, solar power plants have larger floor space so that the data acquisition and display is too fragmented, and there is not a centralized platform for observation, storage of data, resulting in inconvenience and difficulty in analysis and control.
2) Since photovoltaic power station always located in remote area, where the geographical environment and natural conditions are harsh, the station cannot do decentralized maintenance and manual monitoring according to the traditional way [1].

3) Battery in photovoltaic power station is a device with high failure rate. Many reasons can lead to its failure in advance. In addition to unreasonable design, inaccurate maintenance management, unreasonable operation and management of the battery is the main cause [2].

So, the establishment of a control system for the photovoltaic power station is of great practical significance to ensure the safe and reliable operation of the photovoltaic power station. The control system can display electric current and voltage signals from multiple grid-connected photovoltaic power systems centralized in the screen in the field control center. The waveforms at real-time can be displayed at the same time, and the previous data can be checked as well. Remote control center can also read the real-time data on-site to be advantageous for various departments' information sharing, and achieve effective monitoring to the photovoltaic power station. On the basis of studies on intelligent remote monitoring and control system of PV power station, the article proposed a set of building programs of the photovoltaic monitoring system.

\section{RESEARCH STATUS}

Currently, remote monitoring technology applied in the photovoltaic power system can be divided into two types[3] - wired remote monitoring and wireless remote monitoring, according to physical implementation of the means of communication. Wired remote monitoring achieves longdistance signal transmission mainly through communication bus, which signal can be transmitted at real-time, but the distance is limited. The distance cannot be too far even through the repeater. Especially in remote areas, it cannot be achieved to install the system using wired remote monitoring. Wireless remote monitoring is not subject to geographical and distance constraints, especially in remote areas, where this type has special significance.

At present, some popular wired remote monitoring technologies includes: 
1) using the industry bus, such as the 485 bus, CAN bus, etc. To achieve the communication between the lower computer (DSP, MCU, IPC, etc.) and the main monitor PC;

2) linking modem to public telephone network to achieve[4];

3) using combination between Internet and both of the previous methods to achieve. This approach has a wider scope of application and longer distance.

Wireless remote monitoring technology mainly draws support from relaying transmission technology in microwave station or artificial satellite. Such as remote monitoring system which is based on GSM/GPRS wireless mobile communication network, which achieve remote monitoring through the application of data communication services of mobile communications GSM/GPRS or SMS -- Short Message Service and so on. With the $3 G$ mobile communication technology continues to mature and be applied widely, wireless remote monitoring can also achieve mass transfer of data in audio and video. In areas which are non-remote, inconvenient cabling and covered with Internet, combination between Modem and Internet is mainly used to achieve remote monitoring [5].

With the development of information technology, and the achievement of the triple-play (the Internet, telecommunications networks, cable television) technology, it is convenient and fast to achieve remote monitoring through the triple-play technology, which is also the trend of the development of technology of remote monitoring solar photovoltaic power plant.

\section{SYSTEM DESIGN}

\section{A. Technical requirements for system}

The construction of photovoltaic monitoring system should meet principles of safety, reliability, and openness, practical. System should be based on network environment of fiber optic ring, equipment-oriented standard model, and uniform visual interface, and also based on the architecture of security partition.

\section{1) Security}

The operating failure or system defect must not lead to both accidents in photovoltaic inverters, slip case, primary system and the collapse of the secondary system.

\section{2) Reliability}

The important units of the system and the important parts of the units must be redundancy configuration. They can achieve functional reserve by automatically switching to ensure that the availability of system functions are not under the influence of a single equipment failure.

\section{3) Openness}

System should adopt open architecture, providing multitasking and multi-user environment at real-time. Standard of interfaces between modules should be standardized and unified in support of the secondary development of user application software, to ensure that the system can be interconnect and integrated which another system which confirm to relevant standards.

\section{4) Expandability}

System should support online upgrade of extension of functionality, capacity, and is not limited by the original software configuration. The extension of capacity includes the increase quantity of photovoltaic inverter, convergence box linked to the system, and the increase amount of information from the photovoltaic inverter, convergence box, achieving requirements of the system -- overall design, partial implementation.

\section{5) Maintainability}

System maintenance should be guided by principles of simple, convenient and easy-to-use, including three aspects - hardware, software, operating parameters.

\section{B. System architecture}

PV monitoring system is based on six technologies -technology of acquisition and fusion about data from multisystem multi-type, GIS-based technology to process and display the integrated data, technology of information model, software developing technology, network communication technology, and information security technology. The system is divided into four layers: information presentation layer, business function layer, data exchange layer, physical support layer, as shown in Figure 1:

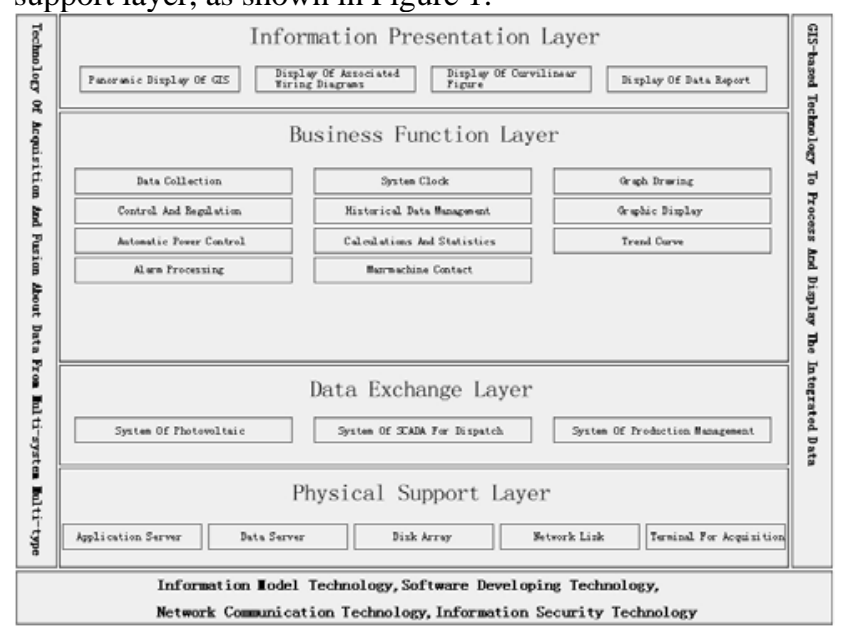

Figure 1. Architecture of the system

\section{1) Physical support layer}

Physical support layer is to provide support at the level of hardware devices on the system, should at least contain an application server for the deployment and publishing of service, a database server used to install the database and data warehouse, a set of RIAD(Redundant Array of Inexpensive Disks) for mass data storage, a set of network link used for data transmission and information exchange, terminal equipment for acquisition used for collection of data, and so on.

\section{2) Data exchange layer}

Data exchange layer is mainly used for data exchange and information-gathering with related business systems, to provide the good data support for the system.

3) Business function layer 
Business function layer is the core of building the system, enabling calculation, analysis of the mass information. As well, this layer establishes the association between different business data, enabling comprehensive analysis and display of integrated multi-system data.

\section{4) Information presentation layer}

Information presentation layer is based on GIS platform in grid. This layer displays the system data visually through combination of field wiring diagram of PV power station, power flow charts and other types of thematic maps, and various curves graphics, reports of data, and other methods of presentation.

\section{SYSTEM FUNCTIONS}

Functions of the monitoring system includes: data collection, control and regulation of photovoltaic inverter, automatic control of power, alarm processing, trend curve, system clock, historical data management, calculations and statistics, man-machine contact, graphic display, graph drawing and so on, as shown in in Figure 2:

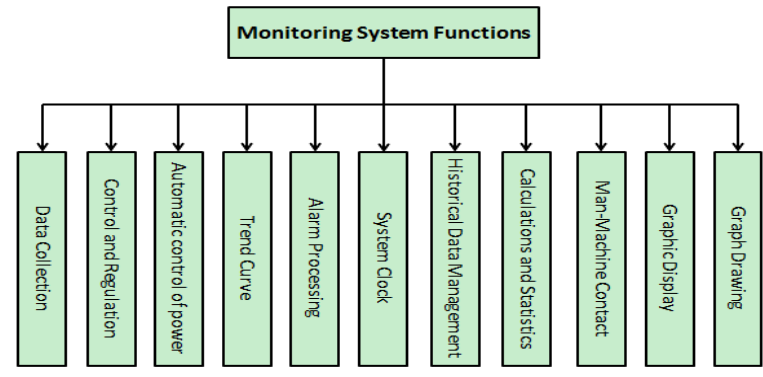

Figure 2. Function structure of the system

\section{1) Data collection}

The system can collect telemetry and teleindication data from photovoltaic inverters, convergence boxes, prefabricated substation, and send all kinds of data information and control commands to the controller at the same time.

\section{2) Control and regulation}

The system can achieve not only the settings of input, output, and power of the photovoltaic inverter, but also remote control of the prefabricated substation.

3) Automatic control of power

Automatic control of power means that the system can track total active/reactive power command of the photovoltaic power plants issued by the joint dispatch, then, make a reasonable allocation to each PV inverters. It also supports emergency command to cut limit power control issued by the joint dispatch.

\section{4) Alarm processing}

As an important part of the system, alarm function should be comprehensive, flexible, practical and intelligent. Alert information should be hierarchical classification to improve accuracy and quickness of accident handling.

\section{5) Trend curve}

Trend curves can show statistical results such as historical, current, planned data, the average, maximum/minimum values of the curves, and the time of emergence, etc.

\section{6) System clock}

System should be able to ensure that the time in all servers, workstations are consistent. There should have security measure for the accuracy of receive clock, and artificial set is available.

\section{7) Historical data management}

Historical data management means that the system saves data regularly, and storage period can be set for the historical data points. Historical database can save data at regular time and store data at abnormal situation.

\section{8) Calculations and statistics}

The system can calculate all the collected data comprehensively, and then, derives new analog value, state value and calculate value, in which the derived calculate value can make a database definition, processing, filing, and calculation just like the collected data.

\section{9) Man-machine contact}

The system should be able to control user permissions to the system through the set of jurisdiction; it should be able to monitor system performance, including the whole system, state of the computer resources, CPU load, network load, memory and disk usage, data transfer speed; it should also visual means of monitoring the system performance, such as trend curve, pie charts, bar graphs.

10) Graphic display

The system should be able to support the capability of full graphics, multi-window. The graphics should be designed as multi-layer, and each layer can display different content and can achieve endless zooming; the system should be able to provide a set of images for system monitoring and control, including system architecture configuration images, system operation monitoring images, system management images.

\section{1) Graph drawing}

Graph editor should have the function to support the establishment of a primitive library of all devices in the system, and can associate with the corresponding records of the device in the database. Also, the user can generate a variety of primitives by themselves.

\section{CONCLUSION}

This paper analyzes the problems the solar power generation and grid-connected PV faced, according to which a proposal about a set of intelligent monitoring system for photovoltaic power plant is presented, which is able to meet the needs of effective monitor to photovoltaic plant in electric power enterprises, and which is of great significance for the safe and reliable operation of the photovoltaic power station. 
[1] Fu Zhongwen, Li-Ping Wu, Yuan-Yuan Han. The development status and prospects of China's photovoltaic industry. China Energy. 2006. 28(6), 9--10.

[2] Chen Huiling. Discussion on measures to extend the battery life of stand-alone PV power station. Battery. 2005. 106--107.

[3] Chen Chao, Remote data communications and network monitoring and management of photovoltaic power station. HeFei University of Technology. 2004.

[4] Gao Song, Ni Yuan, Zhang Hefang. The remote monitoring system based on MODEM. Jornal of Xi'an institute of technology. 2005,25(1): 45 47.

[5] Yang Xianhui. Field bus technology and its applications. Beijing: Tsinghua University Press. 2000. 